\title{
EDITORIAL
}

\section{SPORTS SCIENCE: ENHANCING VERSATILITY IN REHABILITATION SCIENCES}

Being relatively fresh discipline, sports science has gained popularity in the last quarter of the 20th century; it reflects the seriousness with which sports and fitness are approached in the present day. Sports science is quite multidisciplinary field that sightsees the biomechanical, physiological and anatomical variations happening in sportsperson psychological effects to scrutinize the performance nutritional values to significantly prepare the body and also managing the financial and operational needs to enhance the business in sports industry.

Sports science, is a blend of multiple fields that primarily relies on the scientific principles of exercise training and performance. It inculcates the athlete with the biomechanics, anatomy, physiology, psychology, nutrition and many other elements that work in combination to enhance the sports performance.

From the past two decades Pakistan is pushing hard to create a mark internationally in sports industry, although it started approximately 60 years ago. Physical education, Health and Sport sciences have increased resourcefulness in the dynamics of rehabilitation practices. Sports has been an integral part of Pakistani culture, around sixty years back Pakistan sports board (PSB) was created to bring in structural organization and promote fields like cricket, hockey, squash and polo along with the other traditional sports. The role of PSB was only to promote the participation in sports and promote opportunity for sports activities but physical education and sport sciences as subject for education was rudimentary.

The growing trend in sports activities is proportional to health and fitness thus generating demand of advocacy in sports sciences and physical health. To promote sustainability or improvement in the performance, education in sport science has played significant role. In today's era sports is no longer just a leisure but a career, mode of treatment, way of meditating oneself and participation in sports promote socialization too. In short, it covers the physical, mental and social aspects of health.

Sport activities in Pakistan have been increasing since last few years that include participation of both men and women at national and international level but due to lack of resources, technology and experts in sports sciences the goal is yet to achieve. Most of the sports are still in their pre mature developmental phase mainly because of the lack of education, and thus Pakistan is not much successful in competing in international sports events and Olympics. The field of sport sciences is escalating with each passing year so as the need of education.

Ziauddin University, department of Rehabilitation Sciences has taken a lead and under its umbrella along with physical therapy and occupational therapy, physical education, health and sport sciences is also introduced. Physical Education, Health and Sport Science is offered as a discipline in many colleges and universities that encompasses theoretical knowledge as well as hands-on skills along with lab and field work. Ziauddin College of Rehabilitation Science offers four-year degree programme as well as two years associate degree programme. The prime ambition of the programme is to develop and harvest the students in such a way that they would be able to combat with the challenges of the modernization and continuously changing world with a focus on physical performance, activity and health. By going through the process it will enhances the human sporting exhibition at the higher level. During the academic training, students will learn basic science subjects and gain the knowledge regarding kinesiology, philosophical basis of physical education, health and sports sciences, biomechanics, anatomy, exercise physiology, basics of games and gymnastics and later involves the sports training, field work, management and psychological aspects of sports training. In the same course of time student will receive specialize training regarding the injury prevention, enhancing activities and performance and capabilities of the athlete. Specialized field trips and sports rotations will be provided to the graduates to develop versatility.

A graduate from Physical Education, Health and Sports Sciences will see themselves working as an elite sports trainer, coach, exercise and conditioning specialist in sports performance industry, and help the athletes to compete efficiently to achieve new records in various sporting events and battleships.

It has now become the most demanding profession among the health industry. The employment possibilities in this domain has a lot adaptability, from player to coach, sports manager, fitness instructor, sports journalist, athletic trainer, personal coach or an entrepreneur. There is an association between the physical health 
educator and rehabilitative professionals; they may utilize their expertise in Cryotherapy, Thermotherapy, and Electromagnetic Therapy etc. They may work for all age groups across the life span.

Globally as well as locally physical education, health and sport science has multi career opportunity. The lifestyle of modern era human utmost need is to keep themselves not only in good physical shape buy also healthy. Pakistan has Blve Ocean for the career of physical education, health and sport science since it's not been explore yet, all major hospitals, multinational companies, international and local firms are promoting physical educators or sport science graduates to be a part of their team. It's definite that the scope of physical health education and sport sciences has remarkable future in upcoming years with versatility in career field of rehabilitation sciences.

\section{Muhammad Usman Khan}

Assistant Professor / HOD Physical Education, Health and Sports

Sciences

Ziauddin College of Rehabilitation Sciences

Ziauddin University 